\title{
Origin of spatial genetic structure in an expanding oak population
}

\author{
ARNDT HAMPE,*† LEILA EL MASRI*†§ and RÉMY J. PETIT*† \\ *INRA, UMR 1202 Biodiversité, Gènes \& Communautés, 69, Route d’Arcachon, F-33610 Cestas, France, †Université de \\ Bordeaux, UMR 1202 Biodiversité, Gènes \& Communautés, 69, Route d’Arcachon, F-33610 Cestas, France, $¥$ Present address: \\ Department of Integrative Ecology, Estación Biológica de Doñana (CSIC), Av. Américo Vespucio s/n, E-41092 Sevilla, Spain, \\ §Present addresses: Zoological Institute, Evolutionary Ecology and Genetics, Christian-Albrechts-University Kiel, Am \\ Botanischen Garten 1-9, 24118 Kiel, Germany, and Institute for Evolution \& Ecology, University of Tübingen, Auf der \\ Morgenstelle 28, D-72076 Tübingen, Germany
}

\begin{abstract}
Spatial genetic structure (SGS) results from the interplay of several demographical processes that are difficult to tease apart. In this study, we explore the specific effects of seed and pollen dispersal and of early postdispersal mortality on the SGS of a seedling cohort $(\mathrm{N}=786)$ recruiting within and around an expanding pedunculate oak (Quercus robur) stand. Using data on dispersal (derived from parentage analysis) and mortality (monitored in the field through two growing seasons), we decompose the overall SGS of the cohort into its components by contrasting the SGS of dispersed (i.e. growing away from their mother tree) vs. nondispersed (i.e. growing beneath their mother tree) and initial vs. surviving seedlings. Patterns differ strongly between nondispersed and dispersed seedlings. Nondispersed seedlings are largely responsible for the positive kinship values observed at short distances in the studied population, whereas dispersed seedlings determine the overall SGS at distances beyond c. $30 \mathrm{~m}$. The paternal alleles of nondispersed seedlings show weak yet significantly positive kinships up to c. $15 \mathrm{~m}$, indicating some limitations in pollen flow that should further promote pedigree structures at short distances. Seedling mortality does not alter SGS, except for a slight increase in the nondispersed group. Field data reveal that mortality in this group is negatively density-dependent, probably because of small-scale variation in light conditions. Finally, we observe a remarkable similarity between the SGS of the dispersed seedlings and that of the adults, which probably reflects dispersal processes during the initial expansion of the population. Overall, this study demonstrates that incorporating individual-level complementary information into analyses can greatly improve the detail and confidence of ecological inferences drawn from SGS.
\end{abstract}

Keywords: density-dependent mortality, parentage analysis, pollen dispersal, Quercus robur, recruitment, seed dispersal

\section{Introduction}

Seed dispersal and seedling recruitment generate the initial template for plant regeneration and hence can have a great influence on population genetic structure.

Correspondence: Arndt Hampe, Fax: 0034-954-621125;

E-mail: arndt@ebd.csic.es;
Whereas seed dispersal shapes the spatial configuration of sites where individuals establish, early seedling recruitment represents a major demographical filter in the plant life cycle. This stage of the life cycle is characterized by a distinct peak mortality driven by environmental pressure and density-dependent processes (Hamrick \& Nason 1996; Jordano \& Godoy 2002; Wang \& Smith 2002; Howe \& Miriti 2004). Accordingly, 
population genetic studies have demonstrated that dispersal and density-dependent mortality can shape the spatial distribution of genetic variation within populations (Doligez et al. 1998; Epperson 2003; Leblois et al. 2004). Reciprocally, the temporal dynamics of fine-scale spatial genetic structure (SGS) has been used to infer dispersal and other demographical processes (e.g. Hossaert-McKey et al. 1996; Epperson \& Álvarez-Buylla 1997; Kalisz et al. 2001; Jacquemyn et al. 2006, 2008; Jones \& Hubbell 2006; Jones et al. 2006). Limited dispersal is generally considered to be the most common factor involved in the formation of kinship structures, resulting in positive SGS (Epperson 2003; Vekemans \& Hardy 2004), whereas density-dependent mortality, or thinning, is often invoked to explain decreases of kinship structures observed during successive demographical stages (e.g. Epperson \& Álvarez-Buylla 1997; Chung et al. 2003, 2007). Although such studies represent a first step for a better understanding of the interface between the demography and the evolution of populations, they do not distinguish between the different demographical processes involved (e.g. pollination, seed production and dispersal, density-dependent and independent recruit mortality; Wang \& Smith 2002; Jones et al. 2006; Troupin et al. 2006). Hence, unequivocally linking fine-scale spatial patterns of genetic variation to specific processes during plant recruitment remains a challenge.

A few recent studies have used complementary information to address the role of particular demographical processes and their determinants for SGS evolution (e.g. Sezen et al. 2007; Jacquemyn et al. 2008 rate and extent of seed dispersal; Valbuena-Carabaña et al. 2007: level of hybridization; Chung et al. 2007: successional stage; Pardini \& Hamrick 2008: number of founders). The cited studies go beyond traditional descriptive SGS accounts by testing specific a priori hypotheses through the comparison of populations that differ in the character or process of interest. However, their focus at the population level makes it difficult to attribute individual processes, such as dispersal, to SGS.

A more analytical approach would be to gather complementary biological information for the sampled individuals (instead of focusing on the population as a whole) and to examine how individuals that share a given trait contribute to the overall SGS. The focus on individual-level information should help identify the signature of specific demographical processes contributing to SGS while minimizing the influence of confounding effects inherent in the comparison of geographically separate populations. For example, any new cohort of recruits typically consists of individuals that have been dispersed away from their source plants by some vector and others that have failed to be dispersed, and conse- quently form sib aggregations beneath their mother tree. Each of these two fractions of the population should contribute very differently to the overall SGS, and identifying their respective contributions will further clarify the actual effect of dispersal on SGS. A similar logic applies to the effects of postdispersal recruit mortality on SGS: Directly comparing the initial SGS of a seedling cohort with that of the survivors after a given period is likely to provide important novel insights on SGS dynamics. No study to date has used this approach; instead, studies typically compare different age classes without directly assessing the effects of dispersal or mortality on SGS (e.g. Kalisz et al. 2001; Chung et al. 2003; Jacquemyn et al. 2006; Jones \& Hubbell 2006).

Moreover, seed dispersal and postdispersal mortality are intrinsically linked: Dispersed recruits typically have marked higher chances of survival than nondispersed ones, because they are more likely to escape from density-dependent mortality (Hille Ris Lambers et al. 2002) and to arrive at favourable sites for establishment (Wenny 2001). Hence, the rate of densitydependent plant mortality depends on seed dispersal (Harms et al. 2000; Hille Ris Lambers et al. 2002). Novel approaches that couple data on dispersal of individuals and postdispersal mortality into SGS analyses will contribute to our understanding of how both processes interact.

In this study, we investigate the SGS of a pedunculate oak (Quercus robur L.) seedling cohort recruiting within and around an expanding forest stand. We use data about dispersal of plants (deduced from parentage exclusion analyses) and early postdispersal mortality (recorded over two growing seasons in the field) to address the consequences of both processes for the evolution of SGS. This information allows us to unequivocally assign differences in kinship structures of the different groups to the process in question. In particular, we (i) decompose the overall SGS of the cohort into its components contributed by locally recruiting individuals (i.e. those not dispersed away from their mother tree) vs. individuals dispersed more broadly, (ii) derive paternal haplotypes, for seedlings growing beneath their mother trees, to determine the contribution of pollen flow to the kinship structure, (iii) monitor how the initial SGS of the cohort changes through two growing seasons as a consequence of spatial patterns of plant mortality and (iv) compare the SGS of seedlings with that of the adult population. Our goal is to clarify the roles of dispersal and early recruit mortality on the evolution of this particular oak population. Although our SGS estimate refers to realized dispersal (i.e. including early postdispersal recruit mortality) and not to actual dispersal (see e.g. Jordano \& Godoy 2002; 
Jones et al. 2005), sampling seedlings just after their emergence should minimize differences in SGS patterns resulting from all dispersal events.

\section{Methods}

\section{Study area and system}

This study was performed in the Nezer Forest $\left(44^{\circ} 34 \mathrm{C}, 1^{\circ} 00 \mathrm{C}\right)$, located in a large coastal plain, some $50 \mathrm{~km} \mathrm{SW}$ of Bordeaux, SW France. The area is covered by extensive plantations of maritime pine (Pinus pinaster Ait.) interspersed with small (typically $<5$ ha) stands of broadleaf forests dominated by pedunculate oak (Quercus robur L.) and, to a lesser extent, Pyrenean oak (Q. pyrenaica Willd.). Such stands are largely exempt from forest management. Isolated trees or small groups of planted oaks also occur along roads, and an increasing number is naturally recruiting in firebreaks and in the understorey of pine plantations.

We selected an $~ 1.5$ ha mixed oak forest stand containing approximately 280 adult oaks (85\% Q. robur, $15 \%$ Q. pyrenaica) for this study. The stand is surrounded by relatively open plantations of adult pines (tree height: 15-20 m). It is situated on the grounds of a former pine plantation; the abundance and spatial distribution of young oaks within and around the patch clearly indicate that its expansion is still ongoing. In addition to the surrounding pine plantations, there are treeless areas along two firebreaks and on the edges of a small pond (Fig. 1). Understorey vegetation within and around the stand is mostly dominated by bracken (Pteridium aquilinum L.) or blue grass (Molinia caerulea L.); scrub (mostly of Ulex europaeus L., Erica cinerea L., Frangula alnus Mill., and Betula pubescens Ehrh.) is coming up in some parts. The nearest forest fragment (smaller than the investigated one) is approximately $1 \mathrm{~km}$ away.

Figure 1 shows the spatial distribution and the size of adult oaks at the study site. The population consists of one dense patch - the forest stand itself - plus an adjacent, somewhat larger area with lower tree densities. Some large trees grow scattered across the area (within the stand as well as along the firebreak and around the pond visible in Fig. 1a); the smaller trees are likely to be either first or second generation descendants of these few old 'founder' trees. The younger adults, as well as any saplings and seedlings, have presumably established from naturally recruiting acorns.

Most acorn dispersal in the area is performed by common jays (Garrulus glandarius L.), although some dispersal by rodents (European squirrels, Sciurus europaeus L. and wood mice, Apodemus sylvaticus L.) should
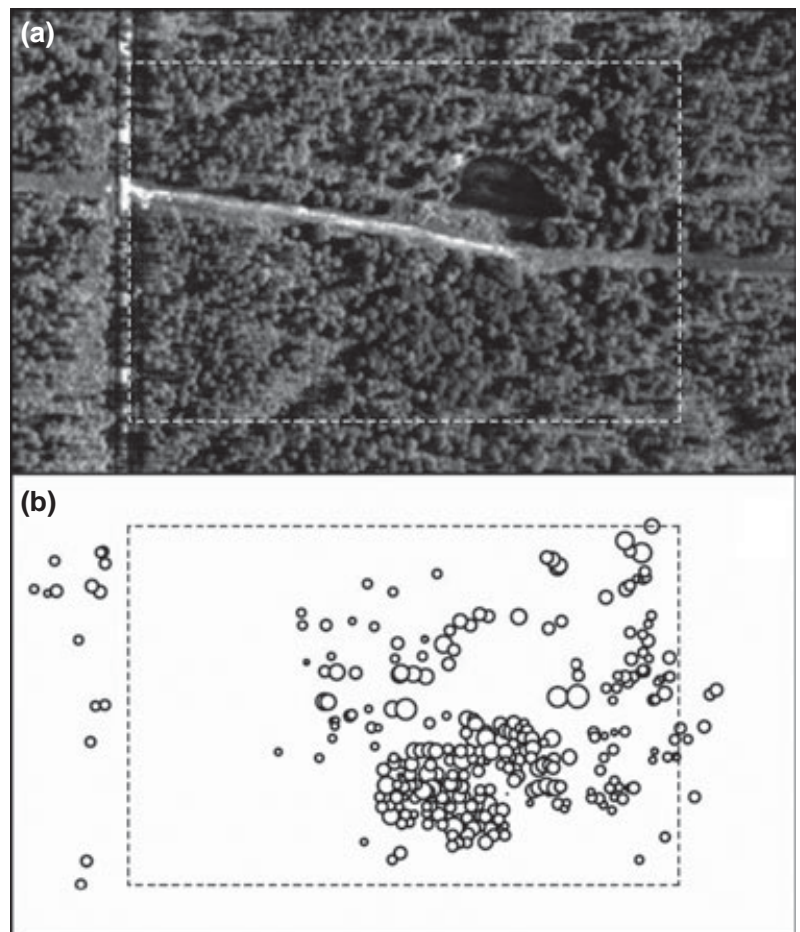

(c)

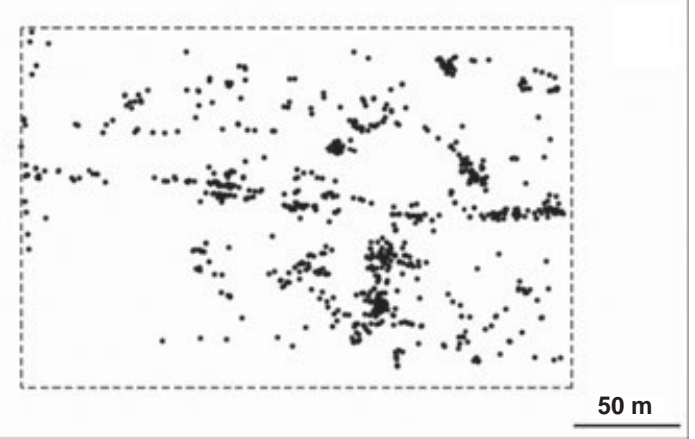

Fig. 1 Aerial photograph of the study area (a) locations of adult oak trees with circle sizes proportional to their d.b.h. (b) and locations of seedlings sampled (c) The broken line indicates the limits of the seedling sample plot. Straight lines without tree cover in the aerial photograph are forest aisles that serve as firebreaks and routes for vehicles, whereas the dark treeless area in the upper right part of the sampling plot is a small pond.

also take place (A. Hampe, personal observation). Jays are very efficient dispersers that can move thousands of acorns within a single fruiting season and regularly transport them over dozens of metres up to kilometres (Bossema 1979; Gómez 2003; Pons \& Pausas 2007). In habitat mosaics such as the one of this study, jays tend to store acorns outside oak stands, showing clear preferences for open areas with low or scarce vegetation or conifer plantations with poorly developed understoreys (Bossema 1979; Kollmann \& Schill 1996; Mosandl \& Kleinert 1998; Gómez 2003). 
Field studies at the site have shown that seedlings growing near adults are on an average attacked more frequently by the rust fungus Microsphaera alphitoides Griffon et Maubl. and by different invertebrate herbivores than seedlings growing away from adult trees. In addition, seedling performance strongly depends on the light microenvironment, which is less favourable beneath oak canopies than in pine plantations or in open areas (A. Hampe, unpublished data).

\section{Field sampling}

We selected a study plot of $\sim 6$ ha enclosing the oak forest stand (Fig. 1). All adult trees within this area and an adjacent belt of $100 \mathrm{~m}$ width were individually tagged and GIS-mapped, and their diameter at breast height was measured. Trees were identified as adults based on their size (i.e. d.b.h. and height) and on fruit set observations performed during two ripening seasons. We collected several buds from each tree and stored them in a freezer at $) 80^{\circ} \mathrm{C}$ for later DNA isolation.

During late April and early May 2006, we performed a comprehensive survey of newly emerged oak seedlings (i.e. those corresponding to the 2005 fruiting episode). For our analysis, we sampled $20 \%$ of all seedlings emerging beneath adult oaks (i.e. up to $\sim 2 \mathrm{~m}$ beyond the projection of their canopy to account for potential short-distance dispersal of acorns by wind or bouncing off of branches). These $20 \%$ were selected using a strict randomization protocol - first estimating the sample size of the overall seedling pool and then determining the seedlings to be sampled based on a random number table - to achieve a representative sampling of the seedling pool. Furthermore, we sampled all seedlings emerging more than $\sim 2 \mathrm{~m}$ away from any adult oaks, searching every part of the study plot at least twice and up to six times. The resulting data set ( $\mathrm{N}=802$ seedlings) includes $25-30 \%$ of the 2006 seedling cohort, forms a representative subset of the overall gene pool and closely reflects the actual spatial distribution of the cohort growing within the sampling plot (except for the different sampling intensity beneath vs. away from oaks). Because Q. pyrenaica seedlings were much less frequent - the sample included only 16 individuals - this species was excluded from subsequent analyses. All seedlings sampled were individually tagged and GIS-mapped, their growth environment was recorded (habitat type, vegetation height and distance to the nearest conspecific seedling), and one leaf was collected and stored at $) 80^{\circ} \mathrm{C}$ until DNA isolation. We visited the tagged seedlings several times through the 2006 and 2007 growing seasons and recorded their survival and performance (growth, damages by pathogens and different herbivores).
Molecular analyses

Total genomic DNA was isolated from deep-frozen leaf samples with the Qiagen DNEasy96 kit for plant tissues. Ten SSR loci were PCR-amplified using two multiplex kits of five loci each: QrZAG11, QrZAG39, QrZAG96, QrZAG112 and QpZAG110, as well as QrZAG5b, QrZAG7, QrZAG20, QrZAG65 and QrZAG87. A detailed description of the primers and PCR procedures is given in Lepais et al. (2006). PCR products were sized on a MegaBACE 1000 96-capillaries automated sequencer (Amersham Biosciences) and scored using the Fragment Profiler version 1.2 (Amersham Biosciences). Three complementary strategies were adopted to minimize the risk of mistyping. First, electrophoregrams were independently scored by two of the authors ( $\mathrm{AH}$ and $\mathrm{LM}$ ) and all divergent scorings were reinspected. Second, when repeating the PCR of loci that had not amplified well in a first attempt, we always included the entire multiplex kit of loci (that is, also those that had already amplified well); this allowed us to repeatedly score almost $18 \%$ of the $\sim 24000$ allelic products characterized in this study. Third, we performed parentage analyses (with Cervus 3.0, Kalinowski et al. 2007; see below for details) and manually checked all those seedlings whose genotype differed by just one allele from their putative parent pair. Although we did not quantify our overall genotyping error rate by systematically including blind samples, the different controls and corrections should have helped achieve a reasonably low level of mistyping (probably well below 1\%). In addition, most of the analyses performed, such as kinship measurements, are known to be robust to genotyping errors (Pompanon et al. 2005; Guillot et al. 2008).

\section{Data analyses}

Classification of individuals. To investigate the effect of seed dispersal on SGS, we classified each seedling either as 'dispersed' or 'nondispersed' (being aware that this distinction is somewhat arbitrary; see Cousens et al. 2008). We considered all seedlings that emerged beneath an adult oak (i.e. up to $\sim 2 \mathrm{~m}$ beyond the projection of its canopy) and had a multilocus genotype that shared at least one allele at each locus with the genotype of that same tree to be 'nondispersed', and all others to be 'dispersed'. A three-step parentage exclusion analysis was used to assign each seedling to one of these two groups. First, we considered all seedlings emerging away from any adult oak as 'dispersed'. Second, we conducted a parentage analysis to identify the parents of the remaining seedlings (i.e. those emerging under an adult oak canopy). We used Cervus 3.0 (Kalinowski et al. 2007) for this purpose with the following 
parameters for the simulation: number of candidate parents $=400$, proportion of candidate parents sampled $=0.75$, mistyping rate $=0.01$ and proportion of loci typed $=0.96$. These parameters correspond approximately to the adult population size and to an incoming gene flow of 25-30\%, a value backed by other studies (see e.g. Jensen et al. 2009). The loci Qr39 and Qr65 were excluded from this analysis because they were characterized by a significant frequency of null alleles (Cervus estimates of $6.9 \%$ and $12.4 \%$, respectively). A seedling was considered 'nondispersed' if it grew beneath a candidate parent tree assigned by Cervus. Finally, we performed a direct comparison of the multilocus genotypes of the remaining seedlings with those of the surrounding trees. Seedlings were also classified as 'nondispersed' if we found that they grew beneath a tree that shared at least one allele at all loci (even if this tree had not been identified as the most likely candidate parent by Cervus); otherwise seedlings were classified as 'dispersed'. For this latter analysis, the loci Qr39 and Qr65 were included, but only when the seedling and parent compared were heterozygotes, because in such cases mismatches cannot be caused by the presence of null alleles. Theoretically, we could have erroneously classified some dispersed seedling as 'nondispersed', if it had been removed from its mother tree and dispersed beneath its father tree; this however seems unlikely.

We then constructed the following seven data sets: (i) all seedlings, (ii) all surviving seedlings (i.e. those individuals alive after two growing seasons), (iii) nondispersed seedlings, (iv) nondispersed surviving seedlings, (v) dispersed seedlings, (vi) dispersed surviving seedlings and (vii) adult trees.

Genetic diversity analysis. We calculated the following measures of genetic diversity for each of the seven data sets: allelic richness $\left(R_{S}\right.$, with the rarefaction size $r$ set to the smallest sample size of the groups included in the analysis), gene diversity $\left[\mathrm{H}_{\mathrm{E}}\right.$, calculated using Nei's (1987) unbiased estimator], and heterozygote deficit $\left(\mathrm{F}_{\mathrm{IS}}\right)$. The loci Qr39 and Qr65 were also excluded from the estimations of diversity parameters because of the presence of null alleles. However, we used them for kinship calculations, because this type of analysis is only slightly affected by the presence of null alleles (e.g. Guillot et al. 2008). Furthermore, we tested for heterozygote deficit relative to Hardy-Weinberg expectations by permuting alleles amongst individuals within samples and using $F_{I S}$ as statistic for comparisons. All diversity analyses were performed using FSTAT 2.9.3 (Goudet 2001). Different groups were subsequently compared with paired t-tests performed over loci, with significance levels Bonferroni-adjusted according to the number of tests (StatSoft 2001).
Identification of major spatial genetic discontinuities in the adult population. Before assessing the fine-scale patterns of SGS, we examined whether major genetic discontinuities exist within the adult oak population that might affect our analyses and resulting biological inferences (Born et al. 2008). For this purpose, we used a spatially explicit Bayesian model implemented in the $\mathrm{R}$ package GENELAND 2.012 (Guillot et al. 2005, 2008). GENELAND uses the geographical locations of individuals as prior information to infer the number of populations present as well as their spatial distributions (delimited by major genetic discontinuities) via simulation of the posterior distribution of parameters by Markov chain Monte Carlo techniques. We performed five independent model runs with 50000 iterations each and a thinning of 50, treating the number of genetic clusters as an unknown variable and using the spatial D-model as prior model for allele frequencies (that is, these were assumed to be mutually independent).

Analysis of spatial genetic structure. We assessed the fine-scale SGS of each of the seven data sets using Nason's kinship coefficient $\mathrm{F}_{\mathrm{ij}}$ (Loiselle et al. 1995), as it displays robust statistical properties (Vekemans \& Hardy 2004). Confidence intervals (95\%) were obtained by permuting individual locations 10000 times. Preliminary tests showed that distance classes of $5 \mathrm{~m}$ over a range of $180 \mathrm{~m}$ provide a high spatial resolution, while guaranteeing sufficient sample sizes to get reliable kinship estimates (according to the recommendations of Hardy \& Vekemans 2002 and Cavers et al. 2005). We also calculated the Sp statistic of Vekemans \& Hardy (2004) and compared different data sets by means of a paired t-test performed over loci where appropriate (see below). Significance levels of these comparisons were Bonferroni-corrected to account for multiple testing.

Kinship coefficients depict average genetic similarities between pairs of individuals, which are usually drawn from a uniform sample without specifying the individuals to be compared. However, some biological aspects can be more properly addressed by specifying certain groups of individuals to be compared (e.g. Hardy \& Vekemans 2001). We did this in two further analyses. First, we pooled all seedlings and adult trees and calculated Nason's $F_{i j}$ for all those pairs of individuals that involved one seedling and one adult. This 'betweengeneration' test allowed us to check whether the SGS of the seedlings can indeed be assigned to limited dispersal of propagules from the adults. Second, we estimated the relative contributions of dispersed and nondispersed seedlings to the overall SGS. The $\mathrm{F}_{\mathrm{ij}}$ values of the pooled seedling cohort represent the weighted average of $F_{i j}$ values generated from pairs of 
dispersed seedlings, pairs of nondispersed seedlings, and pairs including one dispersed and one nondispersed seedling, respectively. We calculated these values separately for each of the three combinations to determine their respective contribution to the overall $F_{i j}$ at a given distance.

Finally, to quantify the role of pollen dispersal on the kinship structure of the investigated cohort, we identified the paternal alleles of each seedling by subtracting the maternal alleles from the seedling genotype for all individuals of the nondispersed seedlings group, following the procedure described in Nakanishi et al. (2009). The resulting paternal haplotype was then converted into a diploid homozygous genotype to calculate $\mathrm{F}_{\mathrm{ij}}$ values. In ambiguous cases, in which both the seedling and its mother tree were heterozygotes with the same alleles, the paternal haplotype was converted into the corresponding heterozygous genotype. Once the paternal haplotypes of seedlings had been reconstructed, their SGS was examined as described above.

All kinship analyses were performed with SPAGeDi 1.2 (Hardy \& Vekemans 2002) except the paired t-tests which were performed with Statistica 6.0 (Statsoft 2001). We did not attempt to infer dispersal distances or neighbourhood sizes from our data because their estimation implies a number of unrealistic assumptions regarding population structure and dynamics (Vekemans \& Hardy 2004).

Density-dependence of seedling mortality. Our field data allowed us to formally test for the density-dependence of seedling mortality. This was performed by means of logistic regression analyses with the distance of each seedling to the nearest conspecific neighbour as predictor variable (log-transformed to meet normality assumptions) and its mortality over the 2 years of study as binary response variable. Analyses were performed on the whole sample as well as separately for the two subsets of dispersed and nondispersed seedlings.

\section{Results}

\section{Patterns of genetic diversity}

We sampled a total of 786 freshly emerging Q. robur seedlings. Based on our parentage exclusion analysis, we classified 324 of these seedlings as nondispersed and 462 as dispersed. Amongst the latter, 104 seedlings (22.5\%) were growing beneath adult oaks and their dispersed status was identified using the genetic analyses. We therefore estimate that the sampling includes c. $20 \%$ of the nondispersed seedlings and slightly more than $50 \%$ of the dispersed seedlings of the cohort (the latter proportion was determined as the weighted aver- age of the proportion of dispersed seedlings sampled beneath and away from adult oaks). After two growing seasons, the number of seedlings originating from nondispersed seeds had decreased to 92 (mortality: 72\%), whereas amongst the dispersed category 253 seedlings survived (45\%). Results of the diversity analysis are summarized in Table 1. Dispersed and nondispersed seedlings have similar levels of allelic richness, gene diversity and inbreeding (Bonferroni-corrected $\mathrm{P} \ddagger 0.05 / 18=0.003$ for all paired t-tests). Diversity estimates did not change through the 2 years of monitoring despite elevated levels of mortality. We also did not detect any statistically significant differences in levels of diversity between seedlings and adult trees.

Detection of genetic discontinuities within the adult population

The Bayesian analysis with GENELAND clearly supports the homogeneous nature of the adult population. All five independent runs indicated one single population as the most likely scenario (posterior density $>0.65$ ), and only one of five runs assigned three of the 252 adult trees to another potential source population. Hence, we found no evidence of major genetic discontinuities within the stand.

\section{Fine-scale spatial genetic structure}

Regression slopes of $\mathrm{F}_{\mathrm{ij}}$ on $\log$ (distance) were different from zero in the seven groups studied $(\mathrm{P}<0.001$ according to 10000 permutations). The SGS of the complete seedling cohort (Fig. 2a) is characterized by a marked peak of $\mathrm{F}_{\mathrm{ij}}$ at the smallest distance interval followed by a notable decrease that becomes less pronounced and remains approximately constant beyond a distance of c. $30 \mathrm{~m}$. The separate analysis of dispersed and nondispersed seedlings reveals that both groups contribute very differently to this overall pattern. The $\mathrm{F}_{\mathrm{ij}}$ of nondispersed seedlings (Fig. 2c) peaks strongly at the shortest distance interval, decreasing rapidly farther away and reaching values around or somewhat below zero at distances beyond $25-30 \mathrm{~m}$. In contrast, the dispersed seedlings (Fig. 2e) present only a small peak in the shortest distance interval and a remarkably linear decrease of $F_{i j}$ with distance across the entire range considered. Finally, the SGS of the adult trees is weak (Fig. 2g). It resembles that of the dispersed seedlings except that the slope of $F_{i j}$ on the logarithm of distance is even less steep, as confirmed by a paired t-test over loci $\left(\mathrm{Sp}_{\text {trees }}=0.005, \mathrm{~S} \mathrm{p}_{\text {dispersed seedlings }}=0.009 ; \mathrm{t}=4.94\right.$, d.f. = 9, $\mathrm{P}<0.001$; Table 1).

The 'between-generation' SGS obtained by analysing pairs including one adult tree and one seedling is strong, 
Table 1 Levels of genetic diversity, individual inbreeding and genetic structuring within different plant groups. The multilocus averages of the following statistics are reported: allelic richness $\left(R_{S}\right)$ (standardized to $N=92$ ), gene diversity $\left(H_{E}\right)$, inbreeding coefficient $\left(\mathrm{F}_{\mathrm{IS}}\right)$ and the Sp statistic of SGS intensity of Vekemans \& Hardy (2004). Differences of $\mathrm{F}_{\mathrm{IS}}$ and Sp from zero were tested using 10000 permutations (Bonferroni-corrected): ${ }^{* * *} \mathrm{P}<0.001,{ }^{*} \mathrm{P}<0.05$, ${ }^{\mathrm{ns}} \mathrm{P}>0.05$. Finally, NN indicates the distance of individuals to the nearest conspecific neighbour in metres (median with lower and upper quartile in brackets)

\begin{tabular}{|c|c|c|c|c|c|c|c|}
\hline & \multicolumn{2}{|l|}{ All } & \multicolumn{2}{|l|}{ Nondispersed } & \multicolumn{2}{|l|}{ Dispersed } & \multirow[b]{2}{*}{ Trees } \\
\hline & Seedlings & 2-year plants & Seedlings & 2-year plants & Seedlings & 2-year plants & \\
\hline $\mathrm{N}$ & 786 & 345 & 324 & 92 & 462 & 253 & 254 \\
\hline $\mathrm{R}_{\mathrm{S}}$ & 15.4 & 15.2 & 14.7 & 14.6 & 15.6 & 15.2 & 15.8 \\
\hline $\mathrm{H}_{\mathrm{E}}$ & 0.788 & 0.790 & 0.775 & 0.767 & 0.794 & 0.796 & 0.794 \\
\hline $\mathrm{F}_{\text {IS }}$ & $0.015^{\mathrm{ns}}$ & $0.016^{\mathrm{ns}}$ & $0.002^{\text {ns }}$ & $0.000^{\mathrm{ns}}$ & $0.022 *$ & $0.017^{\mathrm{ns}}$ & $0.010^{\mathrm{ns}}$ \\
\hline Sp & $0.015^{* * *}$ & $0.015^{* * *}$ & $0.026^{* * *}$ & $0.032 * * *$ & $0.010^{* * *}$ & $0.009^{* * *}$ & $0.005^{* * *}$ \\
\hline NN & $0.9[0.3-2.1]$ & $1.1[0.4-2.6]$ & $0.5[0.2-1.0]$ & $0.4[0.2-0.9]$ & $1.3[0.6-3.1]$ & $1.4[0.6-3.0]$ & - \\
\hline
\end{tabular}

indicating that the seedling genetic structure matches that of the adults. Moreover, it is stronger and decreases more rapidly with distance than that observed for the seedling cohort itself (Fig. 3). This behaviour fits well with our expectations, because parent-offspring kinships are typically higher than half-sib kinships, and the distance between a mother tree and its offspring is on average smaller than the distance between two offsprings of the same tree. Unfortunately, no readily interpretable statistical corroboration of this observed differences is possible because of the inclusive nature of the Sp statistic (cf. Vekemans \& Hardy 2004).

When decomposing the SGS of the overall seedling cohort into its components contributed by dispersed and nondispersed seedlings, we found that $F_{i j}$ values resulting from pairs of nondispersed seedlings determine most of the total SGS up to a distance of $10 \mathrm{~m}$, whereas dispersed seedlings contribute more from 10 to $65 \mathrm{~m}$ (Fig. 4); farther away no clear trends were discernible.

No systematic changes in SGS were observed between the recently germinated seedlings and those that survived two growing seasons (Fig. 2b, d, f), neither for the entire seedling cohort nor for the dispersed seedlings group. In contrast, the SGS of nondispersed seedlings slightly increased through time $\left(\mathrm{Sp}_{\text {all }}\right.$ seedlings $=0.026$, $\mathrm{Sp}_{\text {surviving seedlings }}=0.032 ; \mathrm{t}=3.15$, d.f. $=9, \mathrm{P}=0.01$ ).

Comparisons between paternal haplotypes reconstructed for the nondispersed seedlings group resulted in weak but significant positive kinship values up to 15-20 $\mathrm{m}$ and no further trend beyond this distance (Fig. 5). The autocorrelogram was generally smoother than the one derived from the analysis of the entire seedling genotypes (see Fig. 2c).

\section{Density-dependence of seedling mortality}

The logistic regressions revealed different trends for the three data sets. Both the pooled sample and the dispersed seedlings group did not show any signs of density-dependent mortality (pooled sample: $\mathrm{b}=0.059$, $\mathrm{v}^{2}=1.27$, d.f. $=1, \mathrm{P}=0.26, \mathrm{~N}=755$; dispersed seedlings: $\mathrm{b}=0.046, \mathrm{v}^{2}=0.46$, d.f. $=1, \mathrm{P}=0.50, \mathrm{~N}=439$ ). In contrast, mortality in the nondispersed group was negatively density-dependent, that is, it increased with the distance between individuals and their nearest conspecific neighbour $(\mathrm{b}=) 0.25, \mathrm{v}^{2}=6.1$, d.f. $=1, \quad \mathrm{P}=0.01$, $\mathrm{N}=316)$.

\section{Discussion}

Studying the SGS of plant populations is a potentially useful approach to infer the demographical processes that govern their dynamics, yet ecological interpretations of SGS are burdened with many uncertainties. In this study, we have shown that integrating molecular data with relevant biological information from the individuals sampled (and not only the population as a whole) provides a powerful way to link specific demographical processes with genetic structures. In particular, our analysis enabled us to get detailed insights into the effects of seed and pollen dispersal as well as early postdispersal recruit mortality on SGS, which would not have been accessible otherwise.

\section{Dispersal}

The overall kinship structure of the investigated seedling cohort decreases markedly up to 25-30 m; beyond this distance the decline is considerably less steep and remarkably constant. The changing trend suggests that distinct biological processes contributed to SGS formation within the investigated cohort, although the global analysis provides few clues about their nature. First, using a little-used approach relying on intergeneration kinships, we showed that the kinship structure of the seedlings coincides in space with that of the adults, 

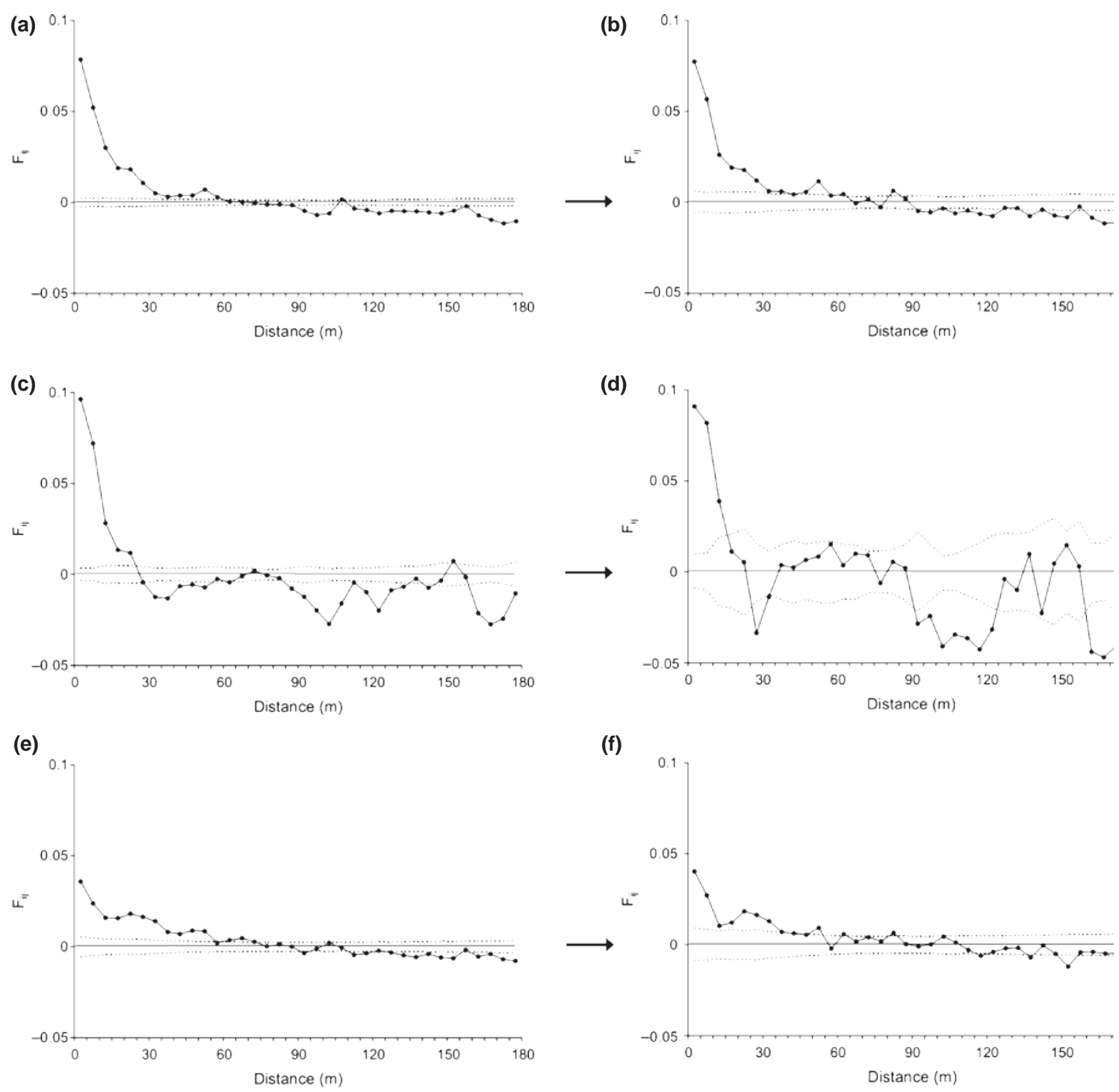

(g)

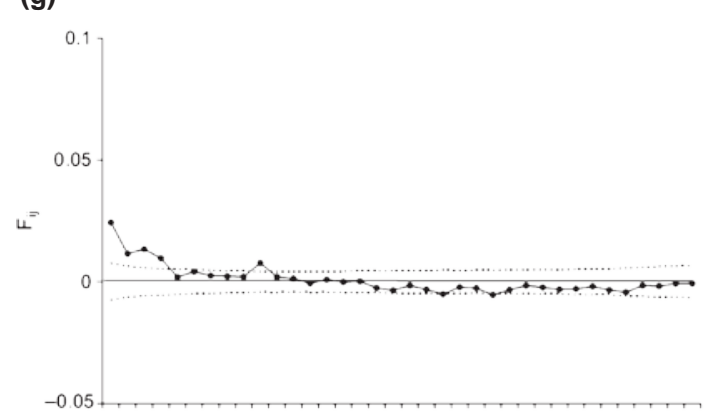

Fig. 2 Spatial genetic structure present in (a) all seedlings, (b) all seedlings surviving two growing seasons, (c) nondispersed seedlings, (d) nondispersed seedlings surviving two growing seasons, (e) dispersed seedlings, (f) dispersed seedlings surviving two growing seasons and (g) adult trees. Broken lines indicate the upper and lower 95\% confidence intervals (10 000 permutations). Arrows indicate evolution after two growing seasons.

demonstrating that dispersal limitations has contributed to build up the SGS of the seedling cohort. Decomposing the overall SGS of the seedling cohort into its components contributed by dispersed and nondispersed seedlings revealed that acorn dispersal plays indeed a central role in shaping the overall pattern observed. The 


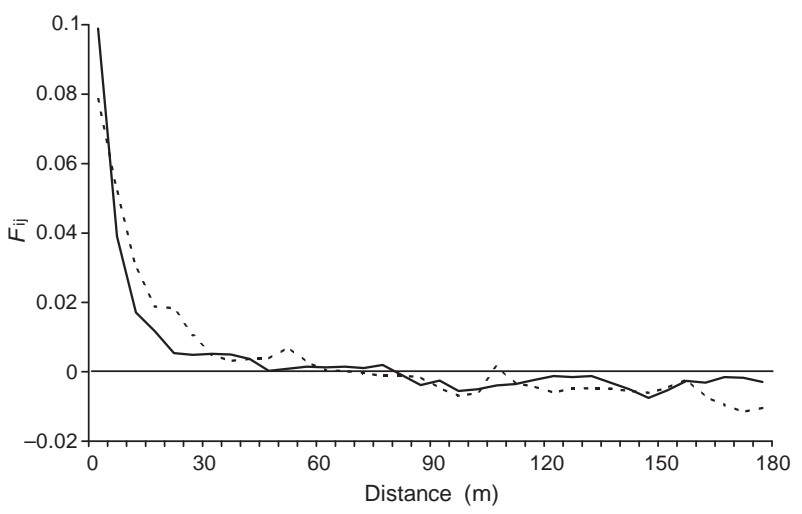

Fig. 3 Plots of kinship values for adult-seedlings pairs (continuous line) compared with seedlings (dotted line). The highly significant 'between-generation' kinship indicates that the SGS of the seedlings can indeed be assigned to limited dispersal of propagules away from the adults. Moreover, the 'between-generation' kinship is stronger and decreases more rapidly with distance than the seedlings kinship, suggesting that it better suited to test for dispersal limitations than kinship measures involving only the seedlings.

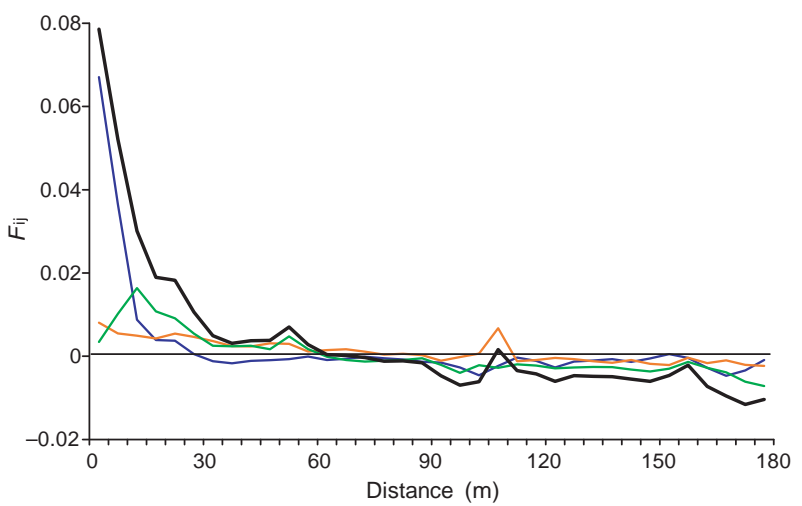

Fig. 4 Spatial genetic structure of the pooled seedling cohort (indicated by the black line), subdivided into its components corresponding to pairs of nondispersed seedlings (blue), pairs of dispersed seedlings (orange) and pairs including one dispersed and one nondispersed seedling (green), respectively.

nondispersed group presents a marked genetic structure in the shortest distance interval $(0-5 \mathrm{~m})$ that diminishes quickly and leaves no apparent trend beyond 25-30 m; in contrast, the kinship coefficient of the dispersed seedlings group declines slightly and almost linearly over the entire distance range. Hence, it appears that the relatively strong signal of the nondispersed seedlings group dominates the overall SGS of the seedling cohort over short distances, whereas the weaker but more far-reaching signal of the dispersed seedlings group shapes it at longer distances. This interpretation is corroborated by the relative contributions of

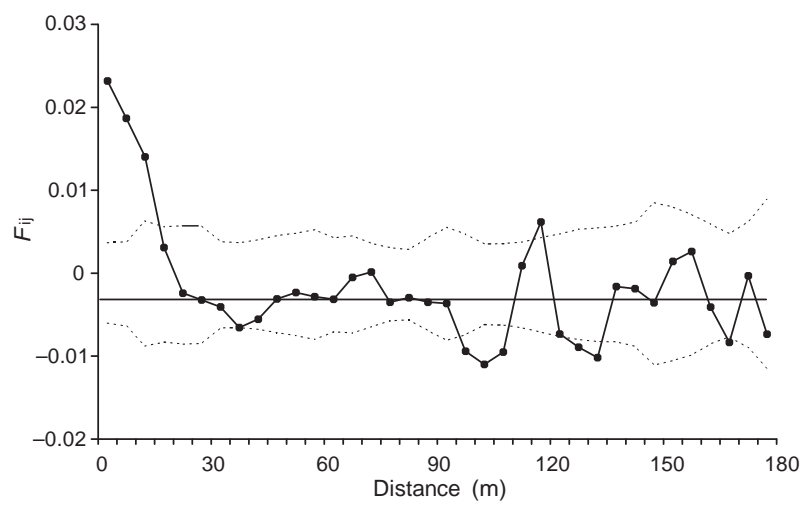

Fig. 5 Spatial genetic structure present in the paternal alleles of nondispersed seedlings. Broken lines indicate the upper and lower 95\% confidence intervals (10 000 permutations).

the dispersed and nondispersed seedlings to the overall SGS of the sampled cohort (see Fig. 4).

Given this strong relationship between acorn dispersal and SGS, it seems necessary to underscore that our analysis is likely to provide a somewhat biased picture of the real whole-cohort SGS. This is because nondispersed seedlings (c. $20 \%$ sampled) were underrepresented in our sampling scheme in comparison with dispersed seedlings (c. $50 \%$ sampled). Hence, the kinship structure of the entire cohort should display an even more pronounced peak at the shortest distance intervals, and the distance-dependent effects described above should likewise be even stronger.

One could have expected that the SGS of the nondispersed seedlings group would reflect that of the adult population at distances farther than the canopy diameter, as adults and nondispersed seedlings share similar spatial locations and genetic composition; yet their kinship structures were very different. This lack of agreement is probably due to the highly skewed contribution of adults to the seedling pool, with a few individuals producing many offspring and many others few or none (the 10 most fecund trees produced in fact $58 \%$ of the nondispersed seedlings). Hence, kinship values beyond c. $30 \mathrm{~m}$ in the nondispersed seedlings group should basically represent statistical noise arising from a sort of pseudoreplication: For a given distance interval, the pairs of seedlings used to compute kinship values will often originate from the same pair of highly fecund genetically (dis-)similar parent trees.

Overall, the results support our expectation that the contribution of individual seedlings to the overall SGS is closely related with their dispersal history (see also Jones et al. 2006; Nakanishi et al. 2009). How did the contrasting kinship structures of dispersed and nondispersed seedlings arise? In the case of the nondispersed seedlings group, two biological processes should be 
influential. The marked genetic structure observed at the shortest distances can primarily be assigned to the important fraction of maternal half-sibs growing in close vicinity beneath their source trees (see Asuka et al. 2005; Nakanishi et al. 2009 for similar cases). This effect should be observable up to the maximum distance covered by adult canopies (around $10 \mathrm{~m}$ ). However, the observed pedigree structure extends several metres farther (see Fig. 2c). Short-distance seed dispersal cannot be involved because of our study design (because all seedlings growing farther than $\sim 2 \mathrm{~m}$ away from the projection of the canopy of their mother trees were considered 'dispersed'). The observed SGS could partly be explained by the slightly positive kinship structure that exists amongst the adult trees (Fig. 2g). Moreover, limited pollen dispersal (i.e. frequent pollen flow between nearby trees) would generate positive SGS at distances exceeding tree canopy. This hypothesis is corroborated by the weak yet significant positive kinships spanning $15-20 \mathrm{~m}$ that we observed for the inferred paternal haplotypes (see Fig. 5). Hence, preferential pollen flow between nearby trees, together with their own genetic similarity (see Fig. 2g), can explain the spatial extent of positive kinship values of the nondispersed seedlings group. It seems noteworthy that significant pedigree structures spanning $20 \mathrm{~m}$ or more are a common phenomenon in closed oak and beech stands (adults: e.g. Bacilieri et al. 1994; Vornam et al. 2004; Jump \& Peñuelas 2007; offspring: e.g. Asuka et al. 2005; Nakanishi et al. 2009). It is most often explained by limited seed dispersal and common mating between neighbours, yet this study is to our knowledge the first that fully teases both processes apart (but see also Nakanishi et al. 2009).

Dispersed seedlings showed a weaker kinship structure, but it spanned longer distances. This pattern provides interesting insights into the behaviour of the main acorn disperser, the jay. Positive SGS tends to form when the seed shadows of different trees do not completely overlap (e.g. Asuka et al. 2005; Jones et al. 2006; Troupin et al. 2006). Given the great mobility of acornforaging jays (Gómez 2003; den Ouden et al. 2005), this is possible only if jays (i) stick to relatively few trees for their harvesting and (ii) disperse many acorns over relatively short distances from the source trees. Field studies (reviewed in den Ouden et al. 2005) suggest that acorn-foraging jays tend to prefer tall trees growing free or along forest edges, probably because these bear large fruit crops. In our population, such trees represent only a minor portion of the adult trees (well below 10\%). Moreover, most of these tall trees have suitable caching sites in their surroundings (in particular along the firebreaks and the pond shore; see Fig. 1), which are commonly used by the jays. Hence, both conditions could be met to a certain extent. In addition, the caching behaviour of jays itself could also generate some local pedigree structures, because birds tend to ingest several acorns from the same adult tree per foraging bout and to store them within a few metres of each other (Bossema 1979; Gómez 2003). This behaviour could in particular account for the (small) peak in kinship values observed at the shortest distance interval (see Fig. 2e). Finally, we cannot rule out the hypothesis that the observed patterns might at least partly result from short-distance seed dispersal by rodents, although acorns collected and cached by rodents usually suffer very high mortality rates (see Gómez et al. 2003; den Ouden et al. 2005 for reviews on the topic).

\section{Postdispersal recruit mortality}

More than half of the monitored seedlings died over the first 2 years of establishment. Given that mortality was markedly higher in the nondispersed group (69\% vs. 44\%), and considering the different plant densities of the two groups (see Table 1), we expected to observe positive density-dependent mortality, yet the opposite was found. This uncommon phenomenon - especially in woody plants (Petit \& Hampe 2006) - is likely to result from the microhabitat conditions under which seedlings established: Most heavily fruiting adults grow close to forest edges, and great seedling agglomerations occur consequently in areas that receive relatively much light, whereas seedlings growing at lower densities in the forest interior are exposed to shadier conditions (A. Hampe, unpublished data). Hence, the early establishment of nondispersed seedlings was apparently triggered by abiotic conditions rather than by direct competition or density-dependent antagonists such as invertebrate herbivores or pathogens. This situation is likely to change with increasing age of the recruits, so the current trend will probably not persist at later stages.

The negative density-dependence of seedling mortality helps explain the slight increase of kinship values observed in the nondispersed seedlings group. Instead, the stability of SGS observed in the dispersed seedlings group agrees with our observation that mortality was not density-dependent in this group. Furthermore, seedling mortality did not alter the levels of heterozygote deficit, indicating that selection against inbred seedlings played a rather negligible role for SGS evolution in the investigated stand.

Overall, this study illustrates how the dispersal of individuals triggers their survival - a fact well known from demographical studies (e.g. Harms et al. 2000; Hille Ris Lambers et al. 2002) - and thus influences SGS beyond the movement of genes itself. On the contrary, 
the observed trends in SGS and our formal analysis of density-dependence of seedling mortality based on nongenetic data demonstrate that density-dependent mortality interferes little at the stage of early plant recruitment in the studied population.

\section{Recruitment and population history}

Although this study focuses primarily on the seedling cohort, it also allows an interesting inference concerning the adult population. A striking similarity exists between the SGS of the adult trees (Fig. 2g) and that of the dispersed seedlings group (Fig. 2e, f). We believe that this similarity is not just coincidental but results from the fact that both generations have developed in a similar context. Most existing adults must have been dispersed over a certain distance to reach the sites where they established, as was the case for the dispersed seedlings group. In addition, these adults are very likely to be the offspring of the same few highly fecund trees in the stand that also contributed most of the analysed seedlings; that is, they probably share the same parents (or at least mothers) with the seedlings that we sampled for this study. Finally, our observation that SGS is little affected by seedling mortality after 2 years of growth is in agreement with the observed stability of SGS between dispersed seedlings and adults. The slightly lower slope in adults as compared with the dispersed seedlings ( $\mathrm{Sp}=0.005$ vs. 0.009) could be accounted for by the fact that the adults, in contrast to the seedlings, probably derive from multiple fruiting episodes (e.g. Chung et al. 2003; Jones \& Hubbell 2006). If our inference is correct, then the SGS of the adult trees would still reflect the initial colonization of the stand.

\section{Concluding remarks}

Studying the SGS of natural plant populations should help infer the demographical processes that govern their dynamics. However, the indirect nature of the approach constrains the precision and confidence of inferences (e.g. Vekemans \& Hardy 2004; Troupin et al. 2006). Hence, unequivocally assigning SGS patterns to specific processes remains a challenging task. This study illustrates that integrating SGS analyses with complementary biological data recorded for the sampled individuals can markedly improve the identification of processes that have shaped the observed SGS patterns. Such complementary data can stem from a diversity of sources; our example uses parentage analyses and demographical monitoring in the field, but in different contexts other factors influencing patterns of gene flow and/or plant recruitment could be investi- gated (e.g. plant gender, flower or fruit morphology, microhabitat characteristics at individuals' growing sites, or levels of herbivore or pathogen damage).

We think that the approach used in this study can help overcome some shortcomings of traditional SGS studies and stimulate further progress in their use for elucidating the dynamics of natural plant populations in realistic landscape contexts. In particular, it could help dissect the natural heterogeneity of genetic structures at the within-population level and identify its demographical and ecological drivers. Finally, the explicit incorporation of nongenetic information at the level of the individual (instead of cohorts or even entire populations) contributes to further bridge the conceptual gap that still exists between traditional SGS studies and modern landscape genetics approaches.

\section{Acknowledgements}

We thank Begoña Garrido and Jean-Marc Louvet for their excellent help with field work and Erwan Guichoux and Olivier Lepais for their advice in the laboratory. Olivier Hardy provided helpful comments for some of the statistical analyses. The genotyping was performed in the Genome-Transcriptome facility of the Functional Genomic Center of Bordeaux, and benefitted from the support of its staff, in particular of Franck Salin, whose help and guidelines are gratefully acknowledged. Insightful comments from Jérôme Duminil, Kimberly Holbrook, Pedro Jordano and three anonymous referees helped improve the manuscript. This work was funded by a Marie Curie postdoctoral fellowship to AH (grant MEIF-CT-2006025383) and an Ms Fellowship from INRA to LEM. RJP was supported by the European Commission Network of Excellence EVOLTREE and the Conseil Régional d'Aquitaine (projet Contribution des feuillus à la multifunctionnalité de la forêt landaise).

\section{References}

Asuka Y, Tomaru N, Munehara Y et al. (2005) Half-sib family structure of Fagus crenata saplings in an old-growth beechdwarf bamboo forest. Molecular Ecology, 14, 2565-2575.

Bacilieri R, Labbé T, Kremer A (1994) Intraspecific genetic structure in a mixed population of Quercus petraea (Matt.) Leibl. and Q. robur L. Heredity, 73, 130-143.

Born C, Hardy OJ, Chevalier MH et al. (2008) Small-scale spatial genetic structure in the Central African rainforest tree species Aucoumea klaineana: a stepwise approach to infer the impact of limited gene dispersal, population history and habitat fragmentation. Molecular Ecology, 17, 2041-2050.

Bossema I (1979) Jays and oaks: an eco-ethological study of a symbiosis. Behaviour, 70, 1-117.

Cavers S, Degen B, Caron H et al. (2005) Optimal sampling strategy for estimation of spatial genetic structure in tree populations. Heredity, 95, 281-289.

Chung MY, Epperson BK, Chung MG (2003) Genetic structure of age classes in Camellia japonica (Theaceae). Evolution, 57, 62-73. 
Chung MY, Nason JD, Chung MG (2007) Effects of population succession on demographic and genetic processes: predictions and tests in the daylily Hemerocallis thunbergii (Liliaceae). Molecular Ecology, 16, 2816-2829.

Cousens R, Dytham C, Law R (2008) Dispersal in Plants: A Population Perspective. Oxford University Press, Oxford.

Doligez A, Baril C, Joly HI (1998) Fine-scale spatial genetic structure with nonuniform distribution of individuals. Genetics, 148, 905-919.

Epperson BK (2003) Geographical Genetics. Princeton University Press, Princeton, New Jersey.

Epperson BK, Álvarez-Buylla ER (1997) Limited seed dispersal and genetic structure in life stages of Cecropia obtusifolia. Evolution, 51, 275-281.

Gómez JM (2003) Spatial patterns in long-distance dispersal of Quercus ilex acorns by jays in a heterogeneous landscape. Ecography, 26, 573-584.

Gómez JM, García D, Zamora R (2003) Impact of vertebrate acorn- and seedling predators on a Mediterranean Quercus pyrenaica forest. Forest Ecology and Management, 180, 125-134.

Goudet J (2001) Fstat, a Program to Estimate and Test Gene Diversities and Fixation Indices, Version 2.9.3. Available at: http://www.unil.ch/izea/softwares/fstat.html.

Guillot G, Mortier F, Estoup A (2005) Geneland: a computer package for landscape genetics. Molecular Ecology Notes, 5, 712-715.

Guillot G, Santos F, Estoup A (2008) Analysing georeferenced population genetics data with Geneland: a new algorithm to deal with null alleles and a friendly graphical user interface. Bioinformatics, 24, 1406-1407.

Hamrick JL, Nason JD (1996) Consequences of seed dispersal. In: Population Dynamics in Ecological Space and Time (eds Rhodes OE, Chesser RK, Smith MH), pp. 203-236. University of Chicago Press, Chicago, Illinois.

Hardy OJ, Vekemans X (2001) Patterns of allozyme variation in diploid and tetraploid Centarea jacea at different spatial scales. Evolution, 55, 943-954.

Hardy OJ, Vekemans X (2002) SPAGeDi: a versatile computer program to analyse spatial genetic structure at the individual or population levels. Molecular Ecology Notes, 2, 618-620.

Harms KE, Wright SJ, Calderón O, Hernández A, Herre EA (2000) Pervasive density-dependent recruitment enhances seedling diversity in a tropical foret. Nature, 404, 493-495.

Hille Ris Lambers J, Clark JS, Beckage B (2002) Densitydependent mortality and the latitudinal gradient in species diversity. Nature, 417, 732-735.

Hossaert-McKey M, Valero M, Magda D et al. (1996) The evolving genetic history of a population of Lathyrus sylvestris: evidence from temporal and spatial genetic structure. Evolution, 50, 1808-1821.

Howe HF, Miriti MN (2004) When seed dispersal matters. BioScience, 54, 651-660.

Jacquemyn H, Brys R, Vandepitte K, Honnay O, Roldá n-Ruíz I (2006) Fine-scale genetic structure of life history stages in the food-deceptive orchid Orchis purpurea. Molecular Ecology, 15, 2801-2808

Jacquemyn H, Wiegand T, Vandepitte $\mathrm{K}$ et al. (2008) Multigenerational analysis of spatial structure in the terrestrial, food-deceptive orchid Orchis mascula. Journal of Ecology, 97, 206-216.
Jensen J, Larsen A, Nielsen LR, Cottrell J (2009) Hybridization between Quercus robur and Q. petraea in a mixed oak stand in Denmark. Annals of Forest Science, 66, 706.

Jones FA, Hubbell SP (2006) Demographic spatial genetic structure of the Neotropical tree, Jacaranda copaia. Molecular Ecology, 15, 3205-3217.

Jones FA, Chen J, Weng GJ, Hubbell SP (2005) A genetic evaluation of seed dispersal in the neotropical tree Jacaranda copaia (Bignoniaceae). American Naturalist, 166, 543-555.

Jones FA, Hamrick JL, Peterson CJ, Squiers ER (2006) Inferring colonization history from analyses of spatial genetic structure within populations of Pinus strobus and Quercus rubra. Molecular Ecology, 15, 851-861.

Jordano P, Godoy JA (2002) The dynamics of frugivoregenerated seed shadows: demographic and genetic effects. In: Seed Dispersal and Frugivory: Ecological, Evolutionary, and Conservation Issues (eds Levey DJ, Silva W, Galetti M). pp. 305-321, CABI Publishing, Wallingford.

Jump AS, Peñuelas J (2007) Extensive spatial genetic structure revealed by AFLP but not SSR molecular markers in the wind-pollinated tree, Fagus sylvatica. Molecular Ecology, 16, 925-936.

Kalinowski ST, Taper ML, Marshall TC (2007) Revising how the computer program CERVUS accommodates genotyping error increases success in paternity assignment. Molecular Ecology, 16, 1099-1106.

Kalisz S, Nason JD, Hanzawa FM, Tonsor SJ (2001) Spatial population genetic structure in Trillium grandiflorum: the roles of dispersal, mating, history, and selection. Evolution, 55, 1560-1568.

Kollmann J, Schill HP (1996) Spatial patterns of dispersal, seed predation and germination during colonization of abandoned grassland by Quercus petraea and Corylus avellana. Vegetatio, 125, 193-205.

Leblois R, Rousset F, Estoup A (2004) Influence of spatial and temporal heterogeneities on the estimation of demographic parameters in a continuous population using individual microsatellite data. Genetics, 166, 1081-1092.

Lepais O, Leger V, Gerber S (2006) High throughput microsatellite genotyping in oak species. Silvae Genetica, 55, $4-5$.

Loiselle BA, Sork VL, Nason JD, Graham C (1995) Spatial genetic structure of a tropical understory shrub, Psychotria officinalis (Rubiaceae). American Journal of Botany, 82, 14201425.

Mosandl R, Kleinert A (1998) Development of oaks (Quercus petraea (Matt.) Liebl.) emerged from bird-dispersed seeds under old-growth pine (Pinus sylvestris L.) stands. Forest Ecology and Management, 106, 35-44.

Nakanishi A, Tomaru N, Yoshimaru H, Manabe H, Yamamoto S (2009) Effects of seed- and pollen-mediated gene dispersal on genetic structure among Quercus salicina saplings. Heredity, 102, 182-189.

Nei M (1987) Molecular Evolutionary Genetics. Columbia University Press, New York.

den Ouden J, Jansen PA, Smit R (2005) Jays, mice and oaks: predation and dispersal of Quercus robur and Q. petraea in north-western Europe. In: Seed Fate: Predation, Dispersal, and Seedling Establishment (eds Forget PM, Lambert JE, Hulme PE, Vander Wall SB), pp. 223-239. CABI Publishing, Wallingford. 
Pardini EA, Hamrick JR (2008) Inferring recruitment history from spatial genetic structure within populations of the colonizing tree Albizia julibrissin (Fabaceae). Molecular Ecology, 17, 2865-2879.

Petit RJ, Hampe A (2006) Some evolutionary consequences of being a tree. Annual Review of Ecology Evolution and Systematics, 37, 187-214.

Pompanon F, Bonin A, Bellemain E, Taberlet P (2005) Genotyping errors: causes, consequences and solutions. Nature Reviews Genetics, 6, 847-859.

Pons J, Pausas JG (2007) Acorn dispersal estimated by radiotracking. Oecologia, 153, 903-911.

Sezen UU, Chazdon RL, Holsinger KE (2007) Multigenerational genetic analysis of tropical secondary regeneration in a canopy palm. Ecology, 88, 3065-3075.

Statsoft (2001) sтAтіsтica (Data Analysis Software System), version 6. Available at: http://www.statsoft.com.

Troupin D, Nathan R, Vendramin GG (2006) Analysis of spatial genetic structure in an expanding Pinus halepensis population reveals development of fine-scale genetic clustering over time. Molecular Ecology, 15, 3617-3630.

Valbuena-Carabaña M, Gonzá lez-Martínez SC, Hardy OJ, Gil L (2007) Fine-scale spatial genetic structure in mixed oak stands with different levels of hybridization. Molecular Ecology, 16, 1207-1219.

Vekemans X, Hardy OJ (2004) New insights from fine-scale spatial genetic structure analyses in plant populations. Molecular Ecology, 13, 921-935.

Vornam B, Decarli N, Gailing O (2004) Spatial distribution of genetic variation in a natural beech stand (Fagus sylvatica L.) based on microsatellite markers. Conservation Genetics, 5, 561-570.

Wang BC, Smith TB (2002) Closing the seed dispersal loop. Trends in Ecology and Evolution, 17, 379-385.

Wenny DG (2001) Advantages of seed dispersal: a reevaluation of directed dispersal. Evolutionary Ecology Research, 3, 51-74.

A.H. is a population and evolutionary ecologist interested in spatiotemporal dynamics of seed dispersal and plant regeneration. L.M. collaborated in the project during her Master and is currently doing a $\mathrm{PhD}$ on host-parasite coevolution in Caenorhabditis elegans. R.J.P is a population geneticist with broad interest in the evolution and conservation of trees. 